\title{
On the Onset of Type I Edge Localized Modes
}

\author{
G. Y. Antar
}

American University of Beirut, Riad El-Solh, Beirut 1107 2020, Lebanon

E-mail: ghassan.antar@aub.edu.lb

\section{S. I. Krasheninnikov}

University of California San Diego 9500 Gilman Dr., La Jolla, CA 92093, USA

\section{P. B. Snyder}

General Atomics P.O. Box 85608, San Diego, CA 92186, USA

\section{R. A. Moyer}

University of California San Diego 9500 Gilman Dr., La Jolla, CA 92093, USA

\section{R. Pugno}

Max Planck Institut für Plasma Physik Association IPP/Euratom, Boltzmannstr 2, 85748 Garching, Germany

\section{S. Gray}

University of California San Diego 9500 Gilman Dr., La Jolla, CA 92093, USA

\begin{abstract}
.
The onset of type I edge localized modes (ELMs) is investigated on the DIII-D tokamak. A fast imaging camera is used with an integration time of $1 \mu \mathrm{s}$ and a time between frames about $15 \mu \mathrm{s}$ continuously recording for a period of $1.3 \mathrm{~s}$. It is observed that the type I ELMs onset starts with a precursor oscillation at the midplane caused by a toroidally rotating localized structure with a spatial scale increasing with time. This is confirmed by the toroidal set of magnetic probes and continues until the filamentary structure(s) strongly interacts with the first wall at the outer mid-plane. This triggers a strong plasma-wall interaction that later spreads to affect the whole scrape-off layer. The properties of the observed localized structure(s) are in good agreement with the ballooning finger structure occurring in the early non-linear phase of the peeling-ballooning instability.
\end{abstract}


In tokamaks, high confinement modes (H-mode) are characterized by a steep pressure gradient at the edge. This leads to the excitation of edge localized modes (ELMs) causing a quasi-periodic and substantial plasma expulsion from the edge into the scrape-off layer (SOL) [1]. Consequently, they may lead to major problems for ITER-like devices [2] mainly due to the large heat load during short periods of time on the plasma facing components. Moreover, the plasma-wall interaction generates neutrals and impurities that could affect the plasma edge and core properties and thus confinement [3].

A significant number of experimental investigations were dedicated to characterizing ELMs properties and the underlying radial particle and energy transport $[4,5,6,7]$. Visible and infra-red imaging has been used to study ELMs $[8,9,7,10]$ where filamentary structures were observed and their properties were studied in detail.

The goal of this article is to study the onset of type I ELMs [11]. By 'onset', we designate the period preceding ELMs crash where a significant amount of the edge plasma density is driven outside the confined zone into the whole SOL [3]. The experimental data show that ELM onset starts with the so-called precursor oscillation which we reveal to be a toroidally rotating localized plasma structure in the form of a coherent precursor filament $(\mathrm{PF})$ at the pedestal 'mildly' interacting with the first wall at the mid-plane. This interaction does not lead yet to the modification of the main plasma parameters profiles as it is the case during ELMs. Around the divertor region, this structure is not detected by the magnetic probes indicating that it is still connected to the main plasma. This observation would be consistent with the ballooning finger structure that appears in the early non-linear phase during models of the peeling-ballooning instability $[12,13,14]$.

The experiment is performed on the DIII-D tokamak where the shots 122322 and 122323 are studied in detail between 1.2 and $2.5 \mathrm{~s}$ when the camera is recording. The plasma current is 1.4 MA and the toroidal magnetic field is 1.9 T. These are lower single null plasmas with major and minor radii respectively 1.76 and $0.6 \mathrm{~m}$. The distance between the separatrix at the outer mid-plane and the wall is $8 \mathrm{~cm}$. The core density is $4 \times 10^{13} \mathrm{~cm}^{-3}$. The plasma reaches H-mode early in the discharge with $8 \mathrm{MW}$ of neutral 
beam heating leading to type I ELMs.

The Phantom V7 camera is used with $1 \mu$ s exposure time and $\Delta t \simeq 15 \mu$ s between frames. About 68,000 images are continuously recorded during each discharge for a period of $1.3 \mathrm{~s}$. The camera is thus not triggered around ELMs and there is no shuffling or 'reordering' of the images. Only $128 \times 128$ pixels are used covering the volume illustrated in Fig. 1 with a 12-bit resolution. The camera, with its $\mathrm{D}_{\alpha}$ interference filter, are installed on port $90 \mathrm{R} 0$, that is, at a toroidal angle $90^{\circ}$ and a poloidal angle $0^{\circ}$ with respect to the mid-plane. Figure 1(a) shows an image from the camera over which we overlay a schematic of the viewed vessel ports. Since it is the $\mathrm{D}_{\alpha}$ emission that is measured, it is important to highlight that ports $15 \mathrm{R} 0$ and $30 \mathrm{R} 0$ do not present components facing the plasma leading to less neutrals emission and thus less light intensity. The $X$ and $Y$ dimensions of the images are determined at $1.4 \mathrm{~m}$ from the camera where the field lines are tangential to the camera view. This spatial resolution, however, decreases with increasing distance to the camera. To analyze the images, an average image in-between ELMs is determined and the subsequent images are subtracted from it leading to $I$, the $\mathrm{D}_{\alpha}$ intensity variation.

In the movies, the first plasma-wall interaction caused by ELMs takes place during the so-called precursor oscillation which is detected by the camera as localized emission of $\mathrm{D}_{\alpha}$ appearing and disappearing in time. This fact, as shown in Fig. 2, strongly suggests that we are not detecting a global perturbation of the plasma which would rather lead to a continuous plasma-wall interaction despite spatial modulation. Global oscillations are expected in the linear phase of plasma edge instabilities growth. For example, the linear phase of the peeling-ballooning instability is predicted to lead to a spatial modulation of the plasma edge at all poloidal angles (see Fig. 1 in Ref. [14]). The localized interaction would thus be more in agreement with the early non-linear phase of an instability growth like the one leading to the onset of one or multiple ballooning fingers $(\mathrm{BF})$ in the peeling-ballooning theory.

The dynamics of the precursor filament is investigated in Figure 2 showing eight images reflecting one in ten frames during the precursor oscillation phase. The following 
cycle occurs: At $t=-1500 \mu \mathrm{s}$, the plasma-wall interaction occurs near the 90R0 port. The perturbation in the light intensity disappears completely $(t=-1350 \mu \mathrm{s})$ to reappear after roughly $300 \mu \mathrm{s}$ at the region around the $15 \mathrm{R} 0$ port at $t=-1200 \mu \mathrm{s}$; this is caused by the lack of neutrals in the region between $90 \mathrm{R} 0$ and $15 \mathrm{R} 0$ as port 30R0 does not possess plasma-facing components. Afterwards, the perturbation moves outside the camera field of view at $t=-1050 \mu$ s to re-appear in the region tangential to the camera at $t=-900 \mu \mathrm{s}$. The perturbation has higher intensity and the structure has a larger spatial extension. Nevertheless, it disappears again as it passes in front of port 30R0 $(t=-300 \mu \mathrm{s})$ to re-appear in the region in front of the camera at $t=-150 \mu \mathrm{s}$. This corresponds to more than a full rotation of the filamentary structure(s). By combining the time needed to make a full rotation and the time to move from port $90 \mathrm{R} 0$ to $15 \mathrm{R} 0$, one can prove that the edge toroidal rotation is taking place in the counter clock-wise direction at about $12 \mathrm{~km} / \mathrm{s}$. This result is in agreement with the charge exchange spectroscopy measuring the plasma toroidal rotation [15] and may be caused by the neutral beams as they have a counter clockwise component. On the other hand, we stress the continuous increase of structure spatial structure with time (compare $t=-1200$ and $t=-150 \mu \mathrm{s})$ which naturally leads to a lesser distance to the wall and thus more plasma-wall interaction. According to the peeling-ballooning theory, the BF should be toroidally rotating since it is predicted that, while it is bulging out of the separatrix at the mid-plane it is still connected to the main (toroidally rotating) plasma. The above results are in agreement with this description.

An independent confirmation of the PF toroidal rotation is obtained using the array of toroidally displaced magnetic probes at the mid-plane measuring $\partial_{t} B_{p} ; B_{p}$ being the poloidal magnetic field. In figure 3, three low-pass filtered signals at toroidal angles 340, 322 and $307^{\circ}$ are plotted along with a zoom on one of the perturbations (the toroidal angle are denoted in the clockwise direction). It can be readily shown that both the direction, in the counter clock-wise direction, and the amplitude, about $12 \mathrm{~km} / \mathrm{s}$, can be determined as the coherent structure perturbs the magnetic probes one after the other.

The coherent structure perturbation of the target region is investigated by 
comparing the magnetic probe signals at the mid-plane and at the target. Figure 4(a) shows $I$ at the plasma edge for three consecutive ELMs presenting precursor oscillations. In (b) and (c) is plotted the magnetic fluctuations at the mid-plane (MPI66M322D) and at the bottom target plate (MPI89B322D) respectively. At the mid-plane, the raw signal plotted in light gray, contains high-frequency contributions that masks the lowfrequency oscillation. An elliptic low-pass filter suppressing fluctuations above $10 \mathrm{kHz}$ is thus applied. The result is shown in the black solid line where now the precursor 'oscillations' are observed and are consistent with the $\mathrm{D}_{\alpha}$ signals of the camera (and the calibrated filterscope not shown here). Remarkably, no signs of oscillations on the target magnetic coils is detected as shown in Fig. 4(c) suggesting that the precursor coherent structure, in this phase, is still connected to the main plasma with closed field lines. This experimental result is in good agreement with the theoretical predictions of the peeling-ballooning instability where, while it bulges out at the mid-plane, the BF still has closed field lines, hence, it should not significantly perturb the divertor target region.

The fact that precursor oscillations are sometimes but not always detected in $\mathrm{H}$ mode plasmas is a consequence of the different time scales involved. If the time it takes the PF to grow and hit the wall is smaller than the time it takes it to perform a full toroidal rotation, then, oscillations cannot be detected. Consequently, in addition to the plasma parameters that determine the instability growth rate and the toroidal rotation, the distance between the separatrix and the wall plays an important role in ELMs behavior as it is experimentally observed.

Figure 5 shows six consecutive images illustrating the ELM's first strong interaction with the wall which follows the precursor oscillation phase. It is straightforward to deduce that this occurs in a localized fashion. The ports without plasma-facing components (see Fig. 1) have lesser plasma-wall interaction and thus lower $\mathrm{D}_{\alpha}$ signals. This effect is particularly visible in the vertical dark region that cuts the PF signature into two horizontal but tilted stripes. As the ELM-wall interaction persists and increases, this area is eventually filled with neutrals and thus $\mathrm{D}_{\alpha}$ light. We emphasize 
the continuity of the ELM-wall interaction in this phase and during the precursor 'oscillation' phase. They both are caused by elongated structures in the toroidal direction, tilted due to the pitch angle, and localized in the poloidal plane. The main difference is their behavior in time where the strong interaction interrupts the periodic process leading to strong plasma wall interaction at the mid-plane and the beginning of the average profile modification.

In order to highlight the various ELM-wall interactions observed by the fast imaging camera, we illustrate in Fig. 6 the first strong plasma-wall interaction for two ELMs with temporal evolution three times faster than ELM \#11. The difference between the slow (Fig. 5) and the fast evolution (Fig. 6) can be caused by the non-linear evolution of the instability leading to the variation in the ELM rise-time. However, one should not exclude an observation artifact linked to (1), there is no preferential a priori position where the ELM ballooning finger strikes the wall in the toroidal direction, and (2), we are viewing part of the plasma that accounts for approximately $1 / 4$ of the total toroidal circumference (see Fig. 1(b)).

In figure 7 four snapshots of different ELMs are shown as they start to strongly interact with the plasma to emphasize the fact that sometimes two or more precursor filaments are detected. The vertical dark area in the light intensity in Fig. 7 is caused by the $15 \mathrm{R} 0$ port. The dark region along the field lines in-between the two fingers is interpreted as caused by the absence of plasma and thus plasma-wall interaction. The appearance of several filaments was also observed in the precursor phase (see Fig. 2). From the multiple fingers signature, one can assess the poloidal mode number of the initial instability assuming that their formation does not affect the properties of the mode numbers [14]. The distance between the filaments at the mid-plane (about $25 \mathrm{~cm}$ ) is in good agreement with the numerical simulation results performed for the DIII-D tokamak [14] with a toroidal mode number $n=10$. The fact that sometimes several filaments are detected as they interact with the wall is in agreement with the numerical simulation where the process non-linearity leads to the onset of one or several localized structures [14]. 
In conclusion, this letter investigates the onset of type I ELMs on the DIII-D tokamak using a combination of fast imaging and magnetic signals. The precursor oscillation phase reported in the literature and here at the mid-plane is shown to be consistent with a localized filamentary structure that is mildly interacting with the first wall while rotating toroidally. No signature is detected at the divertor, strongly suggesting that it is still connected to the closed field region. The increase of its spatial size, detected by both the fast imaging camera and the magnetic signals, is interrupted by the strong plasma-wall interaction at the mid-plane triggering the enhancement of radial transport into the SOL. The present results are consistent with the peelingballooning instability $[12,13]$ which predicts the existence of such structure(s) in the early non-linear phase. Further investigations should continue on the role of particle and heat loads on the mid-plane wall especially as it might be taking the first strong ELMwall interaction; this issue needs to be revisited for ITER-like devices. Furthermore, the spatio-temporal dynamics of other types of ELMs, like type II or III, are to be investigated as a function of plasma parameters while including a more comprehensive set of diagnostics. 
[1] D. N. Hill. A review of DIII-D in divertor tokamaks. J. Nuclear Materials, 241-243:182, 1997.

[2] ITER Physics Expert Groups on Confinement, Transport, Confinement Modelling, and Database. Chapter 2: Plasma confinement and transport. Nuclear Fusion, 39(12):2175-2249, 1999.

[3] M. R. Wade, K. H. Burrell, A. W. Leonard, T. H. Osborne, and P. B. Snyder. Edge-localizedmode-induced transport of impurity density, energy, and momentum. Physical Review Letters, 94(22):225001, 2005.

[4] A. W. Leonard, T. H. Osborne, M. E. Fenstermacher, R. J. Groebner, M. Groth, C. J. Lasnier, M. A. Mahdavi, T. W. Petrie, P. B. Snyder, J. G. Watkins, and L. Zeng. Transport of edge localized modes energy and particles into the scrape off layer and divertor of DIII-D. Physics of Plasmas, 10:1765, 2003.

[5] M. Bécoulet, G. Huysmans, Y. Sarazin, X. Garbet, Ph. Ghendrih, and contributors to JETEFDA workprogramme. Edge localized mode physics and operational aspects in tokamaks. Plasma Phys. Control. Fusion, 45:A93, 2003.

[6] S. Coda, M. Porkolab, and K. H. Burrell. Characteristics of density fluctuations during DIII-D in the DIII-D tokamak. Nuclear Fusion, 41:1885, 2001.

[7] G. Y. Antar. The poloidal distribution of type-iii edge localized modes in mast. to appear in Phys. Plasmas, 2006.

[8] A. Kirk, H. R. Wilson, R. Akers, N. J. Conway, G. F. Counsell, S. C. Cowley, J. Dowling, B. Dudson, A. Field, F. Lott, B. Lloyd, R. Martin, H. Meyer, M. Price, D. Taylor, M. Walsh, and the MAST team. Structure of DIII-D in mast and the implications for energy deposition. Plasma Physics and Controlled Fusion, 47(2):315-333, 2005.

[9] A. Kirk, T. Eich, A. Herrmann, H. W. Mueller, L. D. Horton, G. F. Counsell, M. Price, V. Rohde, V. Bobkov, B. Kurzan, J. Neuhauser, H. Wilson, the ASDEX Upgrade, and MAST Teams. The spatial structure of type-i DIII-D at the mid-plane in asdex upgrade and a comparison with data from mast. Plasma Physics and Controlled Fusion, 47(7):995-1013, 2005.

[10] T Eich, A Herrmann, J Neuhauser, R Dux, J C Fuchs, S Günter, L D Horton, A Kallenbach, P T Lang, C F Maggi, M Maraschek, V Rohde, W Schneider, and the ASDEX Upgrade Team. Type-i ELM substructure on the divertor target plates in asdex upgrade. Plasma Physics and Controlled Fusion, 47(6):815-842, 2005.

[11] H. Zohm. The physics of edge localized modes (DIII-D) and their role in power and particle exhaust. Plasma Phys. Control. Fusion, 38:1213, 1996.

[12] Steven Cowley, Mehmet Artun, and Brian Albright. Detonation: A mechanism for the explosive release of energy in a plasma. Physics of Plasmas, 3(5):1848-1852, 1996.

[13] Steven C Cowley, Howard Wilson, Omar Hurricane, and Bryan Fong. Explosive instabilities: from solar flares to edge localized modes in tokamaks. Plasma Physics and Controlled Fusion, 45(12A):A31-A38, 2003.

[14] P. B. Snyder, H. R. Wilson, and X. Q. Xu. Progress in the peeling-ballooning model of edge localized modes: Numerical studies of nonlinear dynamics. Physics of Plasmas, 12(5):056115, 2005.

[15] W. M. Solomon, K. H. Burrell, P. Gohil, R. J. Groebner, and L. R. Baylor. Extraction of poloidal velocity from charge exchange recombination spectroscopy measurements. Review of Scientific Instruments, 75(10):3481-3486, 2004. 


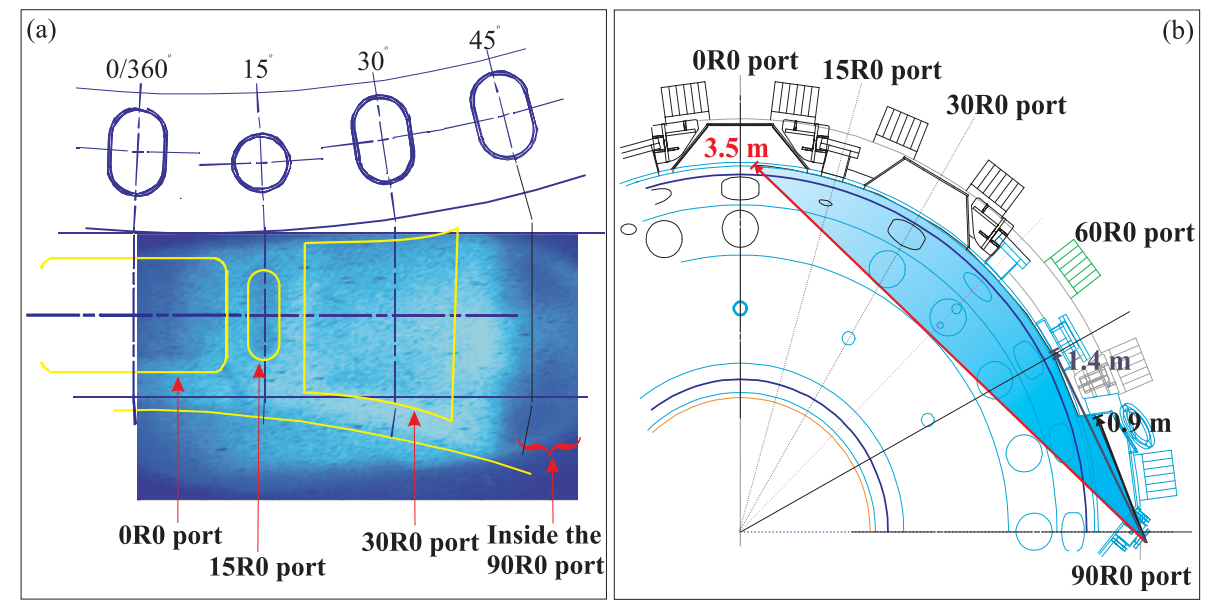

Figure 1. In (a) is illustrated elements inside the DIII-D vessel overlaid on top of a picture from the camera. In (b) is shown a cut through the azimuthal plane. The imaged region is colored with light blue. The distance to where the field lines are approximately tangential to camera view is about $1.4 \mathrm{~m}$. 


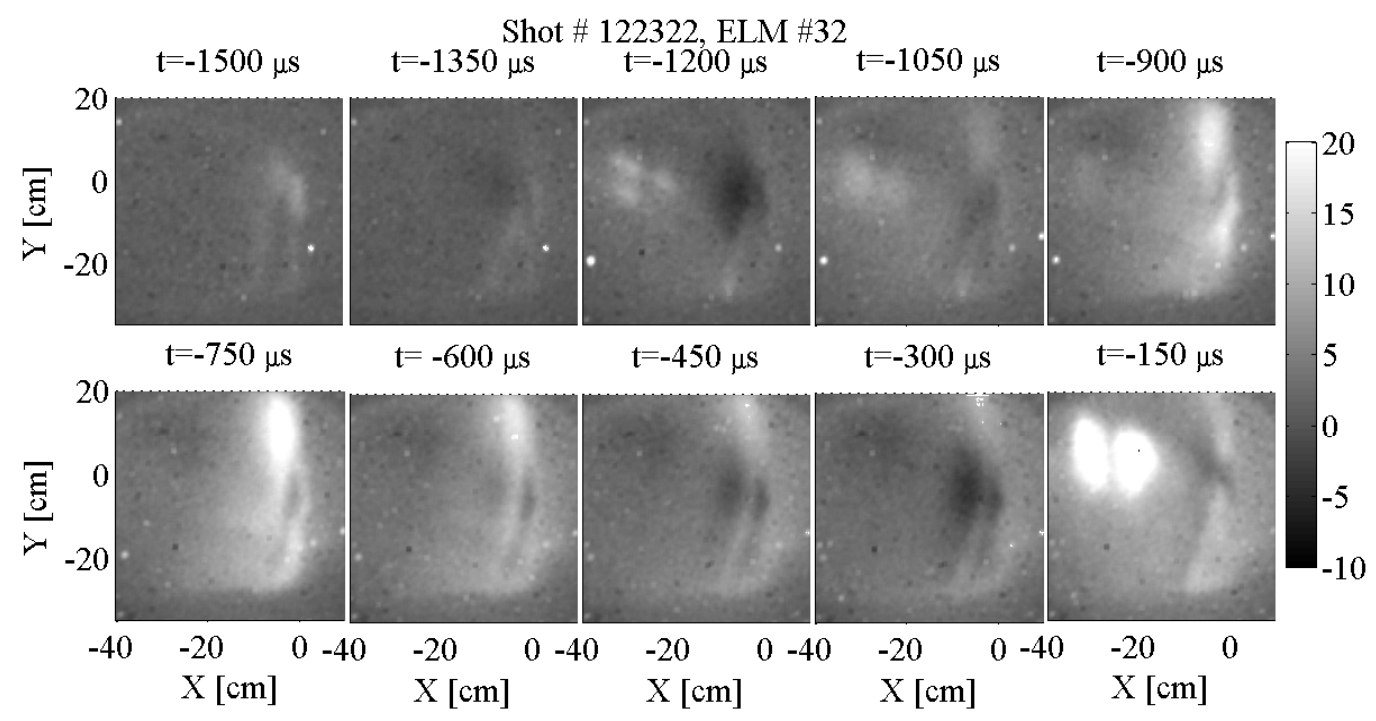

Figure 2. Eight images separated by $150 \mu$ s are illustrated. The same color axis is used. The time $-1500 \mu \mathrm{s}$ is chosen with respect to the ELM crash corresponding to the first strong plasma-wall interaction. 


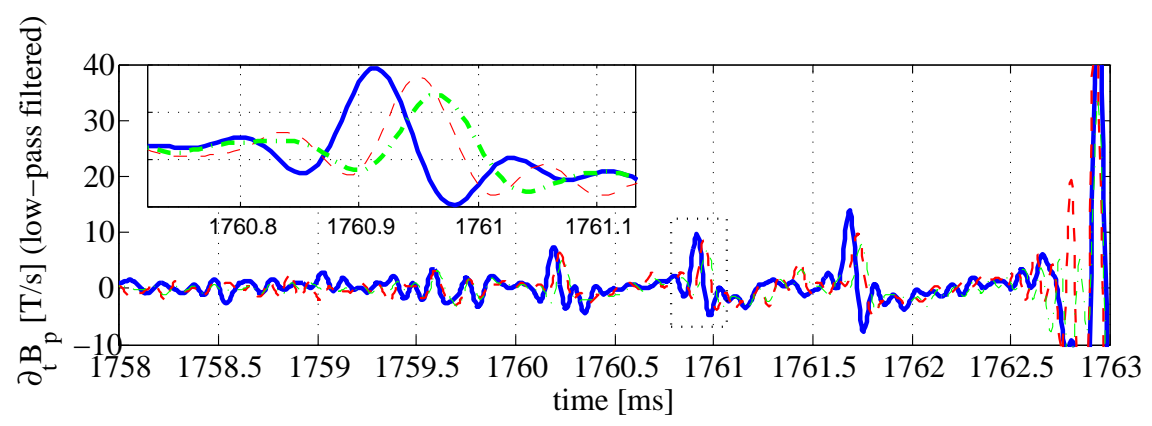

Figure 3. The solid, dashed and dash-dotted lines represent respectively 3 midplane magnetic probe signals (MPI66B307D, MPI66B322D and MPI66B340D) at the toroidal angles 307,322 and $340^{\circ}$. A low-pass filter was applied with a cut-off frequency equal to $10 \mathrm{kHz}$. A zoom is also plotted in order to highlight the time delay among the signals due to toroidal rotation. 


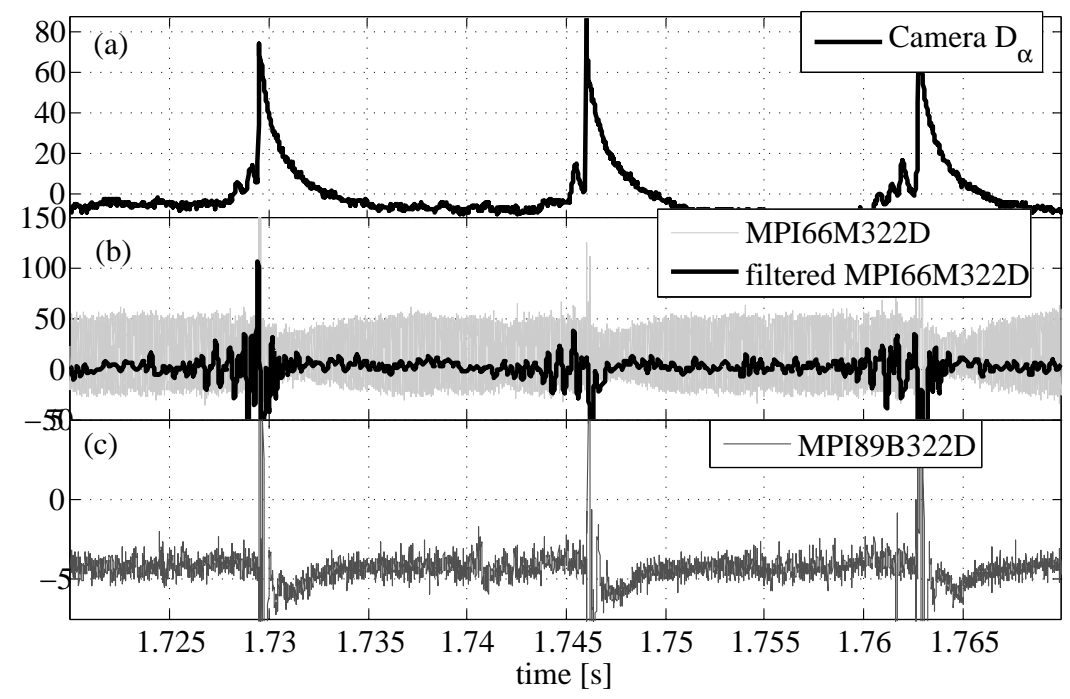

Figure 4. (a) shows the light intensity fluctuations from the fast imaging camera at $Y=0$ and $X=-5 \mathrm{~cm}$ as a function of time. In (b), the gray line represents the magnetic fluctuation at the mid-plane at a toroidal angle of $322^{\circ}$ while in black is the signal after being low-pass filtered. In (c), the target magnetic fluctuations signal (MPI89B322D) is plotted with no signs of precursors. 


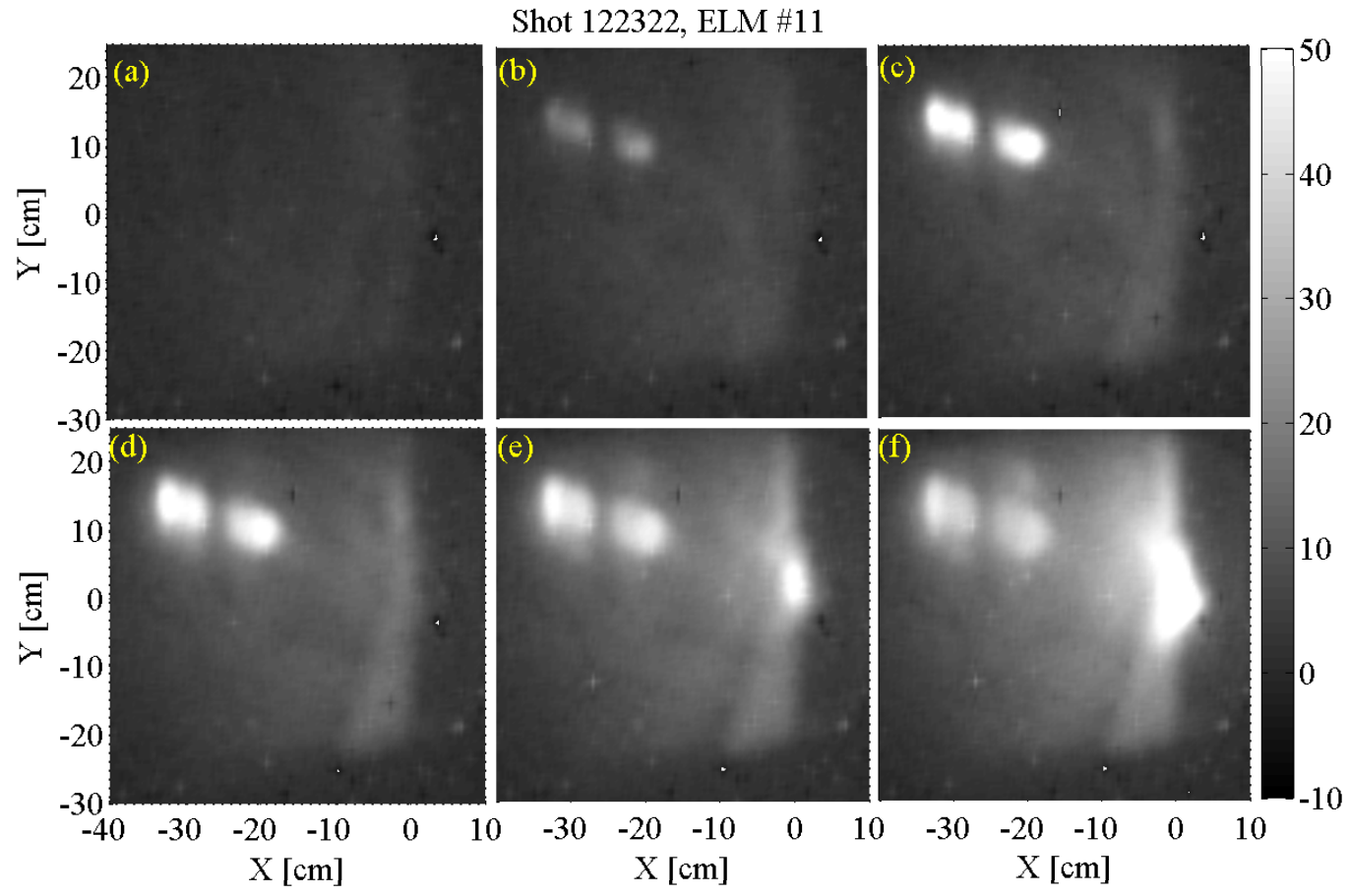

Figure 5. (a) to (f) shows the spatio-temporal evolution of ELM \#11 in shot 122322; the time between frames is $15 \mu \mathrm{s}$. The filament impact is split into two patches by the 15R0 port. For details about the view refer to Fig. 2. 


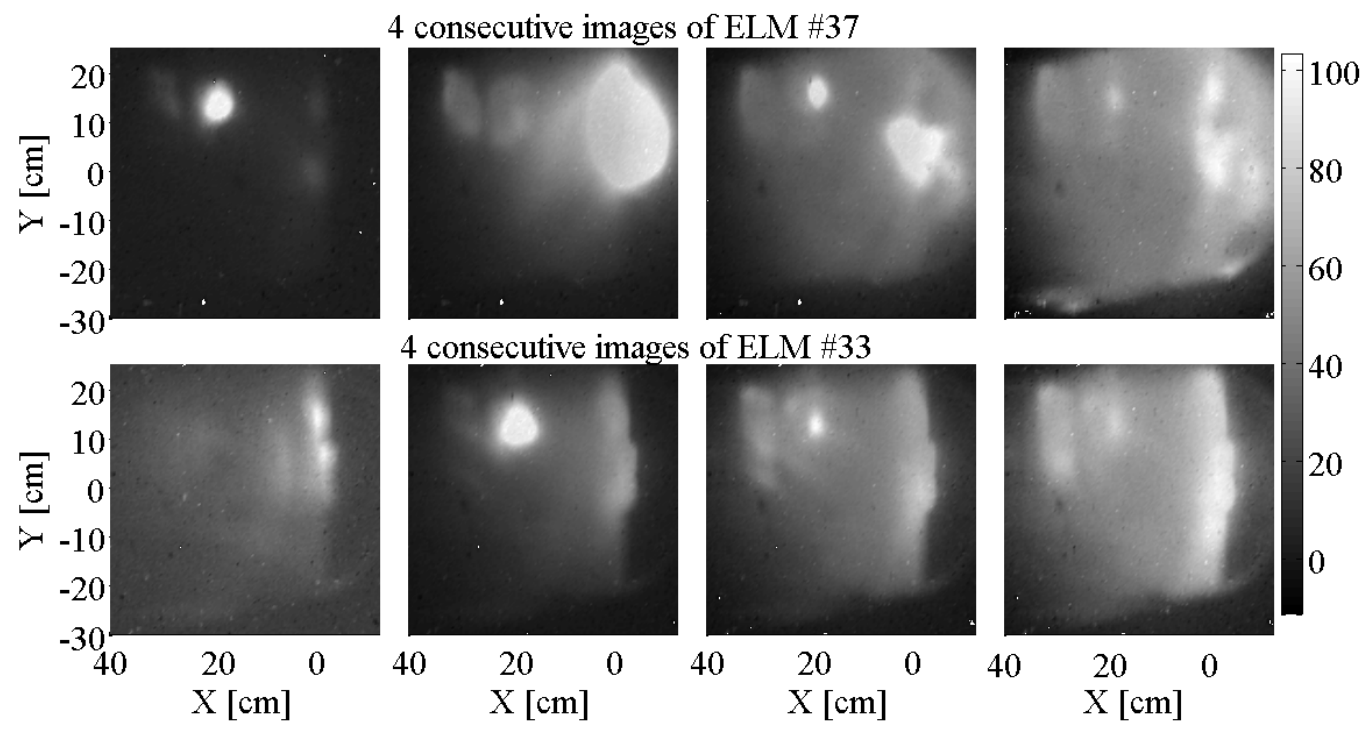

Figure 6. The 'fast' evolution of two ELMs \#37, 33 for four consecutive times (from left to right) is illustrated. 


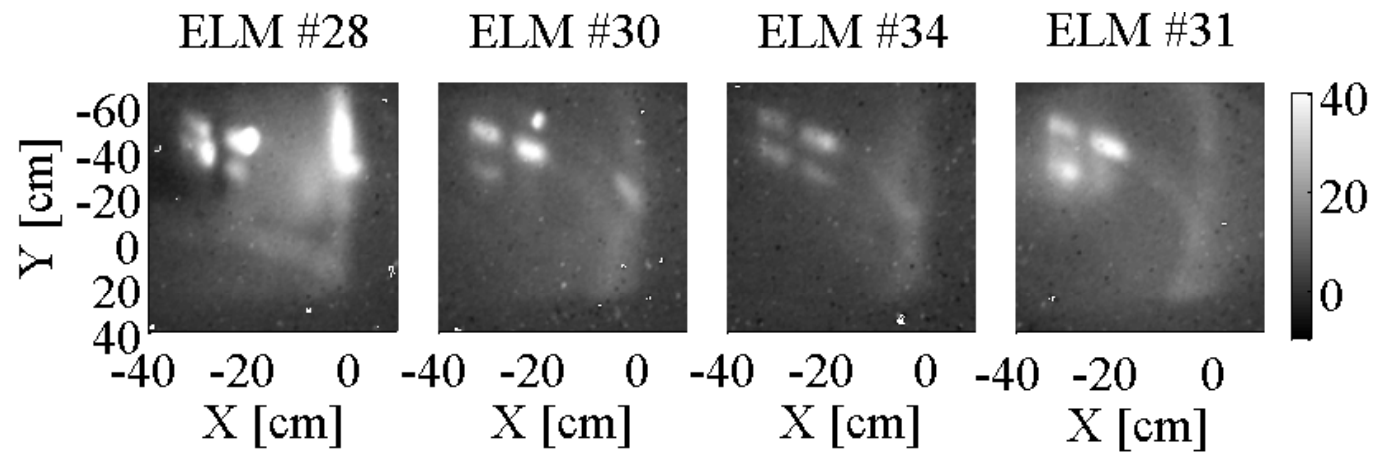

Figure 7. The four images illustrate snap-shots at times around the first strong impact of ELMs with the first wall for four different ELMs. It clearly shows the existence of multiple fingers that are not magnetically connected. The false color is the same for the 4 sub-plots. 